\title{
SALT AND WATER IN THE TREATMENT OF DIABETIC ACIDOSIS
}

\author{
BY DAVID M. KYDD \\ (From the Department of Internal Medicine, Yale University, New Haven)
}

(Received for publication June 30, 1933)

In a previous paper (1) the nature and causes of the bicarbonate deficit and other serum electrolyte changes in diabetic acidosis were discussed from a theoretical standpoint, and disordered electrolyte patterns actually observed were analyzed in the light of this theoretical discussion. It was pointed out that displacement of bicarbonate from combinations with base by ketone acids is a process which can be reversed completely with the contribution from outside sources of nothing except the ability to burn carbohydrate. Base in combination with ketone acids may be considered potential bicarbonate. On the other hand, the loss of base with ketone acids in the urine introduces an irreversible process, because base for the replacement of that sacrificed must come from external sources. Such base deficiences may become quite extreme, but ordinarily they are small compared with the deficiences of base combined with chloride. The actual extent of the body chloride losses is hardly evident from the concentrations of this ion found in the serum, because it is masked by contractions of the body fluids. Such a contraction brought about by loss of water in excess of dissolved salts will increase the concentration of chloride more than it will that of bicarbonate, because the body fluids contain more chloride than bicarbonate. In observed cases, the actual reduction of chloride concentration in the serum usually exceeded that of bicarbonate plus undetermined acids (which for convenience was called $S$ and represents the actual plus potential bicarbonate mentioned above). If loss of body fluids is taken into consideration it is obvious that the actual loss of chloride must far exceed that indicated by the reduction in concentration. The deficiency of base, of course, exceeds that of either chloride or $S$, because it represents the sum of the two base fractions. In some instances, with $S$ well preserved, the reduction of chloride concentration exceeds that of base. This has been interpreted as evidence that the chloride ion, instead of the bicarbonate ion, has yielded base for the neutralization of ketones, a reaction which would seem to be neither useful nor chemically possible. It was pointed out that if equal amounts of base and chloride were excreted and the body fluids were at the same time diminished by a disproportionately large elimination of water, the reduction in concentration of chloride would exceed that of 
base, because contraction of fluid volume would increase base concentration more than chloride. It was suggested that this is a more likely explanation of excessive chloride deficits than the previous theory that the chloride ion is excreted with ammonia to provide base for the neutralization of ketones.

The present paper is intended to analyze the clinical and therapeutic implications of these theories in the light of observations made in a series of patients during recovery from diabetic acidosis. The clinical material consists of twenty-five patients on whom studies of blood sugar together with total acid-base equilibrium of the serum were made at frequent intervals by the methods usually employed in this department (2). In certain instances urine was analyzed for total base or chloride or both. The intakes of fluid, sodium, chloride and glucose were usually measured. Records of output are seldom so complete, because urine specimens and vomitus were frequently lost. Of these twenty-five patients, thirteen are discussed below. The remaining cases so closely resembled those reported that the inclusion of additional data does not seem warranted.

In the discussion that follows it is assumed that insulin and carbohydrate were given to all cases with the intention, at least, of reestablishing glucose combustion and thereby eliminating ketosis as rapidly as possible. If this aim is realized, base previously combined with the ketone acids will be released to form bicarbonate. There can be no difference of opinion concerning the objective of therapy in this respect. Differences in the means of securing this objective will be discussed in another connection. The chief concern of the present study is the nature and quantity of fluids and salts which it is advisable to give. For the most part treatment actually employed has been the administration of large amounts of sodium chloride and fluids together with carbohydrate. When patients have been able to take these materials by mouth the sodium chloride has been given chiefly in the form of non-nutritive broth containing 1 per cent of this salt, the carbohydrates as sweetened drinks containing about 10 per cent of sugar. In addition, liberal amounts of water have been permitted. When oral feeding was impossible 0.9 per cent sodium chloride and 5 per cent glucose solutions have been given subcutaneously or intravenously, and occasionally stronger glucose solutions intravenously. The salt solution is intended to restore the volume of the body fluids and to remedy salt depletion. Glucose solution serves two purposes. 1. It provides carbohydrate for combustion and replenishment of glycogen stores. 2. It offers a medium for the parenteral administration of water without salt. Normal salt solution provides sodium in concentration approximately equivalent to that of total base in body fluids. In respiration and insensible perspiration there is a continuous wastage of fluid without any appreciable quantity of salt. Unless the kidneys, then, excrete base in concentration exceeding that of 
body fluids, administration of normal saline alone would raise base in the body fluids to an abnormally high level. To obviate this difficulty, in addition to the saline, enough water should be given to provide for losses by extrarenal channels. Glucose solutions answer this purpose because the glucose can be oxidized, leaving only the water.

The analysis of the data presented is complex not only because of the large number of variables with which it is necessary to deal, but also because the serum analyses are concerned with concentrations of substances and give no direct information as to total amounts of fluids and solutes in the body. In a preceding article the average compositions of body fluids in normal subjects and patients with diabetic acidosis were compared (1). In Figure 1, diagrams $A$ and $B$ represent schematically

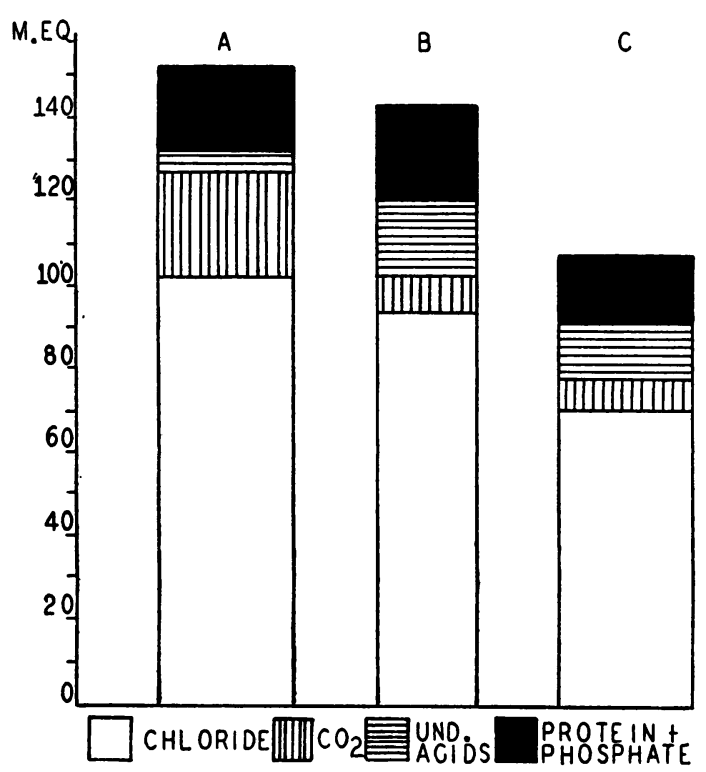

Fig. 1. Diagrammatic Representation of the Chief Electrolyte and Water Changes that Occur in Diabetic Acidosis

The width of columns $B$ and $C$ represents roughly the total body fluid volume compared to the normal $A$.

the two conditions. As far as concentrations are concerned they are quite realistic because all values are derived from observed data. The 25 per cent reduction of body fluid in $B$ represents little more than a guess, because the actual extent of fluid losses during the development of diabetic acidosis has not been established. Diagram $C$ represents the electrolyte concentrations of $B$ reduced by dilution to the original fluid volume found in $A$. Thus $C$ represents the actual salt losses found in diabetic acidosis. It emphasizes the necessity of having some measure 

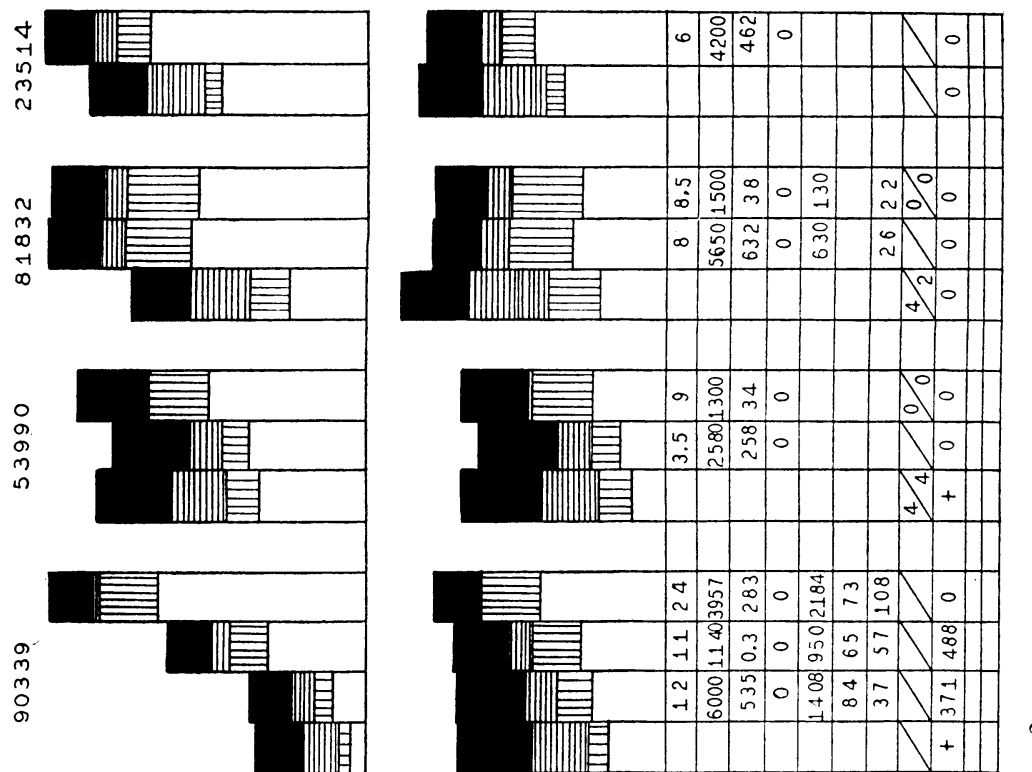

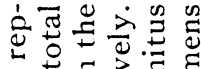

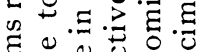

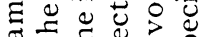

क क क क क क क

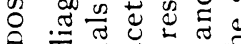

ป ป

$\langle$ 可

- $\Xi$ ๘

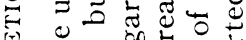

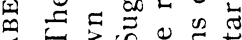

๘

ค

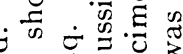

云过吉吉远

当

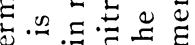

$\approx$ 过

II ठ

O

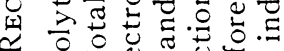

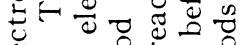

范

$\stackrel{0}{0}=0$

¿ ن

i)

०

$\stackrel{2}{\prime} 00$

(n)

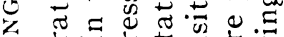

द

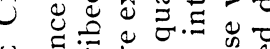

(1)

$>$ \& \&

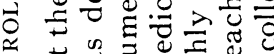

艺的寻

บ Ð

空

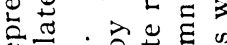

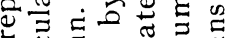

r

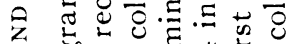

ব

《

.

ن

四 으원휴

$\cong+\infty$ bo

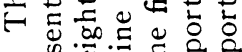

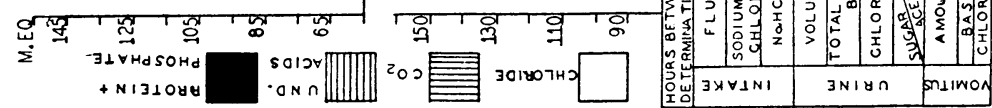

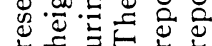



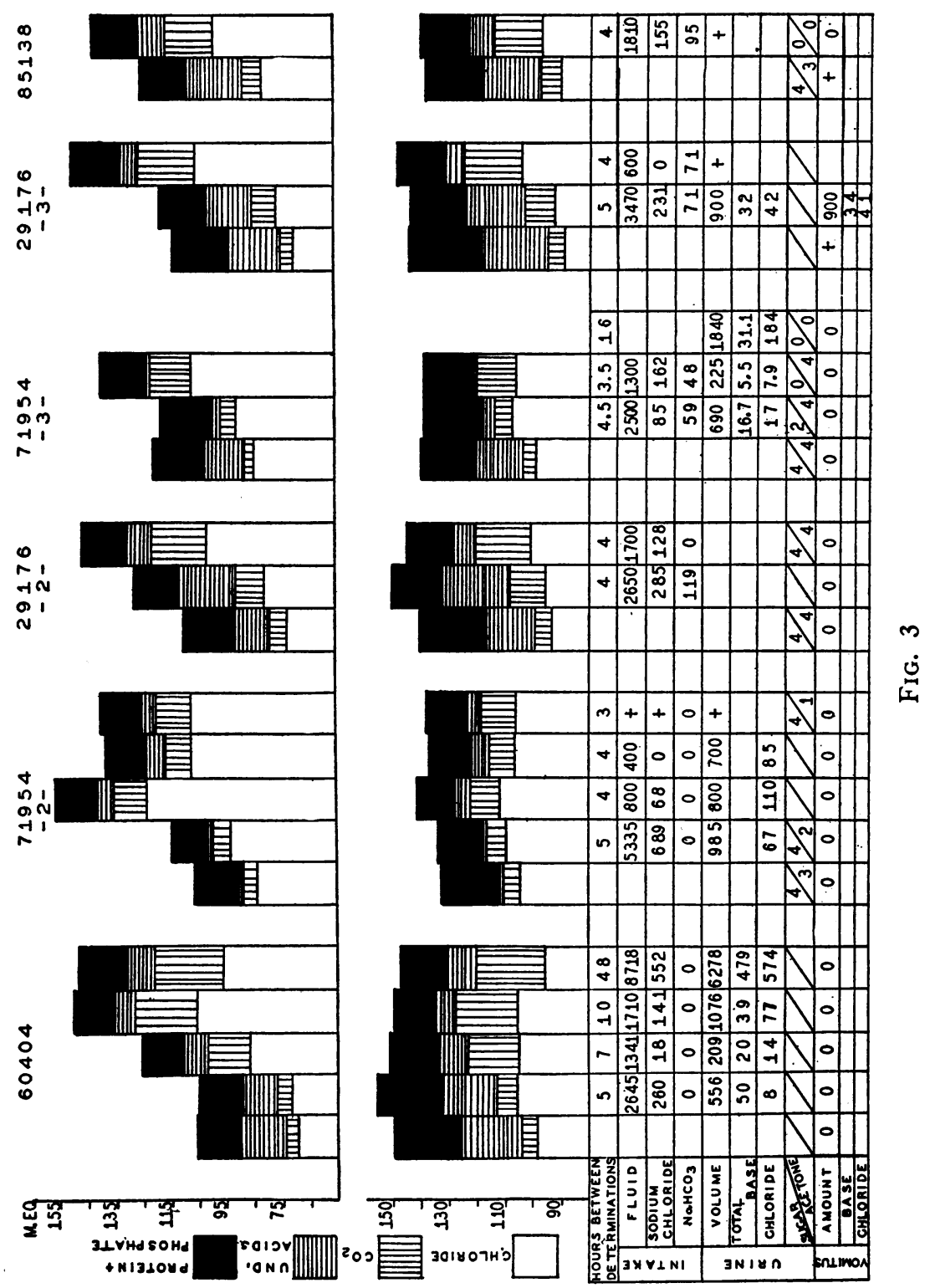
of dehydration in order that electrolyte concentrations determined after various forms of therapy may be adequately compared with the initial concentration. In a previous paper (3) the relation of the serum proteins to hemoconcentration has been discussed. It was pointed out that, except in the presence of shock, the fall of serum proteins from their initially high values probably is associated with, even if it is not exactly proportional to, restitution of body fluid. In the absence of a better and more exact method, the drop in the level of the serum proteins has been used in the succeeding discussion as one measure of body fluid change. Knowing the fluid and salt balances of an individual during a period of time, the changes that occur in the serum electrolyte concentrations afford another measure of body fluid change, if isotonicity of all the body fluids be assumed. It is only by correlating concentrations of electrolytes with changes in body fluid volume that the extent of the original depletion of the salts and their replacement during recovery become apparent.

The analytical data obtained in thirteen of the cases investigated are presented in Figures 2 and 3. The bottom row of diagrams in these figures represents the serum electrolyte concentrations found. Total base is not shown, but equals the total height of the acid column in each case. The top row of diagrams presents the data recalculated to take into account water change. This was accomplished by using the changing serum protein concentrations as an index. For example, in Case 60404, the initial serum protein concentration was $18.9 \mathrm{~m} . \mathrm{Eq}$. and the final, $13.9 \mathrm{~m}$. Eq. Assuming that no protein was lost or gained, the serum volume must have increased 35.9 per cent. All the electrolyte concentrations found at the time of the first determination have been reduced 35.9 per cent in order to eliminate changes in concentration due to differences in the amount of water present. Thus the initial chloride concentration of $98.2 \mathrm{~m}$.Eq. becomes $69.3 \mathrm{~m}$.Eq. Each determination has been compared to the final one in order that the approximate amounts of each ion added could be ascertained. The amount of salt in the intake and in the urine and vomitus are expressed in milliequivalents. Chloride was given in the form of sodium chloride, so that equal amounts of sodium and chloride were given.

In analyzing the data, not only must the effect of changing body water content on the concentration of the electrolytes be evaluated, but also the results of vomiting and nausea must be ascertained. Unfortunately, vomiting, or at least nausea, is one of the most frequent events during the course of diabetic acidosis. Individuals who are vomiting are obviously losing from the body varying amounts of salt and fluid. They have in the intestinal tract a large volume of fluid which, although isotonic with the body fluids in general, may be of entirely different composition in regard to particular ions. Fluid placed in the gastro-in- 
testinal tract eventually becomes isotonic. This is true not only in individuals who are actually vomiting but also in those who are nauseated. When, during recovery, the gastro-intestinal symptoms diminish, this fluid is reabsorbed into the mass of body water. These facts explain otherwise incomprehensible alterations in salt concentrations and fluid balances, observed during recovery, which are not related to the amount of salt and fluid immediately taken into the body or excreted. Case 29176-1 furnishes a qualitative example of these changes. In the first eight hours the patient received $2050 \mathrm{cc}$. of fluid with $154 \mathrm{~m}$.Eq. of sodium chloride. Throughout this time he was vomiting or was nauseated. The water content of the blood was apparently maintained, but base and chloride concentrations diminished; $S$ remained constant. In the next 10 hours his nausea disappeared. Although he received only $850 \mathrm{cc}$. of fluid with no salt during this period and passed $1820 \mathrm{cc}$. of urine, there was about a 10 per cent blood dilution with a marked rise of serum chloride. The concentration of base in the serum remained the same, but as the recalculated diagram shows, the total amount of base actually increased. The most reasonable explanation of these changes seems to be that fluid was first poured out into the alimentary canal, and then reabsorbed. This subject forms a part of a general discussion of water balance to be reported by Lavietes, Kydd and Peters (4).

In order to test the effect of varying therapy the individuals have been divided into four groups. 1 . The patients who were treated with insulin and carbohydrate alone. 2. The patients who were given fluids in addition. 3. The patients who received varying amounts of salt in addition to the therapy used in 1 and 2. 4. The patients who received bicarbonate as well as salt, fluid, carbohydrate and insulin. It has already been stated that the proportions of insulin and carbohydrate used will not be discussed in this paper.

\section{Treatment without salt and fluids}

Case 48711-1 was given enough fluid (as carbohydrate drinks) barely to equal the amount of urine that was formed. She received no salt. Initially she had a rather high undetermined acid in the serum, presumably mostly ketones, while $S$ was only moderately reduced. With this form of therapy the body fluids evidently became somewhat more contracted, as the total base and chloride rose slightly in the first five hours. The ketones rapidly diminished, but $S$ also decreased. Therefore, although its excretion was not measured, it can be inferred that base was excreted. $\mathrm{CO}_{2}$ rises because of loss of fluid and also because of the base liberated from ketones and not excreted. During the next five hours the patient received slightly more fluid, but still an insufficient amount. $S$ is maintained, the undetermined acid fraction is still further reduced and $\mathrm{CO}_{2}$ augmented by an equal amount. In brief, dehydration 
is not overcome and the increase of $\mathrm{CO}_{2}$ that is observed is due to replacement of the ketones that have been burned and to fluid depletion. The excretion of chloride is very small, $3 \mathrm{~m} . \mathrm{Eq}$. Although base excretion was not quantitatively determined, the ketones in the urine, evidenced by a heavy nitroprusside reaction, probably carried base with them. Depletion of electrolytes must have continued throughout the period of study in this individual, but its effects on the serum were masked by the simultaneous loss of fluid. The increase of bicarbonate consequently gives a speciously favorable impression of the efficacy of a therapeutic course which was obviously unsatisfactory if its aim was the restoration of a normal internal environment.

\section{Treatment with fuids alone. (Cases 81042, 29176-1)}

There are no cases that received fluids entirely without salt. The cases listed in this group received less salt than they excreted. Case 81042 was given $2120 \mathrm{cc}$. of fluid and only $46 \mathrm{~m}$.Eq. of chloride during a period of eighty-two hours in which she excreted $975 \mathrm{cc}$. of urine containing $86 \mathrm{~m}$.Eq. of chloride. No figures for the excretion of base are available. The serum proteins drop from 8.09 per cent to 6.92 per cent, evidencing adequate hemodilution. The $\mathrm{CO}_{2}$ concentration rises because of displacement of ketones. Total base and chloride fall, not only because dilution takes place, but because chloride at least was lost in the urine. Base falls farther than chloride because, since its concentration is higher in the serum, it is more affected by dilution. This phenomenon is discussed in detail elsewhere. This therapy causes dilution of the electrolytes. Any augmentation of $\mathrm{CO}_{2}$ which occurs is referable only to the base liberated by burning ketones. Again, consideration of bicarbonate alone gives an erroneous impression of the results of therapy, and especially of its effects on base.

3. Treatment with varying amounts of salt and fuid. (Cases 90339, 53990, $81832,23514,60404,71954-2)$

This has been the accepted mode of treatment and most of the cases reported fall within this group. The diagrams of individuals exhibit responses of great variability because of differences in initial electrolyte and fluid depletion, the proportions and amounts of salt and fluid given, the amount of vomiting and nausea and the quantities of fluid and salt excreted.

Case 90339 had in the serum an initial chloride concentration of $97 \mathrm{~m}$. Eq. with a total base of $142.8 \mathrm{~m}$.Eq. During the first twenty-two and three-quarter hours she received $(4792 \mathrm{cc}$.) more fluid than she excreted. Her salt intake was $548.3 \mathrm{~m} . \mathrm{Eq}$. of sodium, $28.8 \mathrm{~m} . \mathrm{Eq}$. of potassium and $535.2 \mathrm{~m} . \mathrm{Eq}$. of chloride. She excreted $90.7 \mathrm{~m} . \mathrm{Eq}$. of sodium, $51.6 \mathrm{~m}$.Eq. of potassium and $93.7 \mathrm{~m}$.Eq. of chloride. With this 
therapy the serum chloride concentration rose to $106 \mathrm{~m}$.Eq. Serum $\mathrm{CO}_{2}$ rose because bicarbonate replaced the ketones that were burned and $S$ also increased slightly. This is not easily apparent from the concentrations observed, but becomes so when the extent of original fluid loss, which is represented in the diagram, is considered. During the ensuing twenty-four hours she received $1873 \mathrm{cc}$. of fluid more than she excreted, together with $286 \mathrm{~m}$.Eq. of sodium and $283 \mathrm{~m}$.Eq. of chloride, of which she excreted 52 and $107.8 \mathrm{~m}$.Eq. respectively. At the end of this time her chloride had risen to $118 \mathrm{~m}$.Eq. notwithstanding further dilution. Base was now normal, $150.2 \mathrm{~m}$.Eq. $\mathrm{CO}_{2}$, however, had only risen 3 m.Eq. and was still very low, 17.8 m.Eq. Despite the distorted electrolyte pattern observed at the end of the study, the patient, aside from exhaustion, had felt entirely well for more than twenty-four hours.

Case 53990 on admission was vomiting profusely and was markedly dehydrated. Vomiting and nausea ceased soon after therapy was instituted. During the first three and one-half hours he received $2580 \mathrm{cc}$. of fluid with $258 \mathrm{~m}$.Eq. of sodium chloride. Although the ketones diminished, $\mathrm{CO}_{2}$ fell, presumably because of the continued heavy excretion of the former together with a certain amount of base. In the recalculated diagram it is observed that $S$ fell $8 \mathrm{~m}$.Eq., whereas total base fell $4.8 \mathrm{~m} . \mathrm{Eq}$. and chloride rose $3.4 \mathrm{~m}$.Eq. The amount of sodium chloride added to the serum was apparently insufficient to replace the base and ketones lost. During the next nine hours very little sodium chloride was given, $34 \mathrm{~m}$. Eq. Despite slight retention of fluid, chloride and total base rose rapidly in a parallel fashion. $\mathrm{CO}_{2}$ was increased by further elimination of ketones. Because of the low intake during this period these changes can be interpreted only as the result of absorption of fluid from the gastro-intestinal tract.

Case 81832 was also given large amounts of salt and fluid. In this case salt depletion was not extreme. The effect of the treatment was to cause dilution of the serum with a marked increase of base and chloride. If the observed changes in the electrolytes in this case are recalculated with consideration of the dilution indicated by the diminishing serum proteins, it is found that equal amounts of sodium and chloride have been retained. The increase in $\mathrm{CO}_{2}$ has been almost entirely at the expense of ketones. In Case 71954-2 the initial serum chloride is high, but base is very low, as are the undetermined acids. In the course of thirteen and one-quarter hours he received $4010 \mathrm{cc}$. of fluid more than he excreted and retained $505 \mathrm{~m}$.Eq. of chloride. The large amount of fluid given caused extreme dilution of the serum. Base slowly rises. Chloride, after an initial rise, returns almost to its original concentration. Unfortunately, no base excretion figures are available; but, as $S$ slowly increases, chloride must have been excreted in equal or greater amounts than base. Because chloride concentration in serum is greatly less than base an excretion of 
equal amounts of each will cause a relative increase in serum base concentration.

The administration of both sodium chloride and water permits restoration of base and body fluids. Hyperchloremia often results and bicarbonate may remain low. However, these abnormalities of the electrolyte pattern do not seem to have any deleterious effects as far as one can judge from objective and subjective clinical signs.

\section{Treatment with fluid, sodium chloride and sodium bicarbonate. (Cases 29176-2, 71954-3, 85138, 29176-3)}

Sodium bicarbonate was given to four cases, but no conclusive results were obtained. Case 29176 during the first four hours received 119 m.Eq. of sodium bicarbonate, $285 \mathrm{~m}$.Eq. of sodium chloride and $2650 \mathrm{cc}$. of fluid. Serum base rose from 141 to $151 \mathrm{~m}$.Eq., chloride from 93.2 to $95.6 \mathrm{~m} . \mathrm{Eq}$. $S$ rose from 23.8 to $36.3 \mathrm{~m}$.Eq., but $\mathrm{CO}_{2}$ rose only from 6.7 to $12.0 \mathrm{~m}$.Eq., indicating a very considerable increase in the undetermined acids. During the next four hours no more bicarbonate was given. Further dilution took place. Base concentration fell, as did $S$, but the undetermined acids were greatly reduced, so that $\mathrm{CO}_{2}$ rose. The chloride concentration rose to normal, $101.8 \mathrm{~m}$.Eq. These changes become more comprehensible in the upper figures, where body fluid changes are taken into consideration. It is then apparent that during the first four hours $\mathrm{CO}_{2}$ increases because of an accession of total base. During the second four hours $\mathrm{CO}_{2}$ replaces the ketones and total base and chloride are added in about equal quantities. The reason for the increase in ketone acids after bicarbonate administration is obscure. It is known that bicarbonate will increase the excretion of ketones and in this case qualitative ketones persisted in the urine for a longer period than was expected. It is conceivable that the ketones came from the cells in response to the addition of base to the extracellular fluids.

In Case 71954-3, $107 \mathrm{~m} . \mathrm{Eq}$. of sodium bicarbonate, $247 \mathrm{~m} . \mathrm{Eq}$. of sodium chloride and $3800 \mathrm{cc}$. of fluid were given in eight hours. He developed a hyperchloremia and the concentration of $\mathrm{CO}_{2}$ rose only by virtue of the base liberated by oxidation of ketones. This patient was vomiting on admission and nausea continued for more than three hours. That he then absorbed from his stomach considerable free hydrochloric acid, part of which had been neutralized by the small amount of bicarbonate given, would provide a plausible explanation of the data. In Case 29176-3 much the same phenomena occur. In the last period of four hours $71 \mathrm{~m}$.Eq. of sodium bicarbonate without any chloride were given. During this four hours chloride and base rose. The concentration of $\mathrm{CO}_{2}$ increased from 10.8 to $20.5 \mathrm{~m}$.Eq., but the concentration of undetermined acids fell from 20.7 to $6.6 \mathrm{~m}$.Eq., so that $S$ actually diminished. The sodium bicarbonate given was more than neutralized 
by the contents of the gastro-intestinal tract while the individual was nauseated. The vomitus secured for analysis contained $33.6 \mathrm{~m}$.Eq. of total base and $40.4 \mathrm{~m}$.Eq. of chloride. Case 85138 , in four hours, received $1810 \mathrm{cc}$. of fluid, $155 \mathrm{~m}$.Eq. of sodium chloride and $95 \mathrm{~m}$. Eq. of sodium bicarbonate. $S$ remained constant. The recalculated diagram shows that equivalent amounts of total base and chloride were added to the serum. Undoubtedly, sodium bicarbonate was not given in sufficiently large amounts to produce a demonstrable specific effect upon serum bicarbonate.

\section{DISCUSSION}

The cases are complicated by differences in initial salt and water depletion, the occurrence of vomiting or nausea, and variation in renal function, as well as by the type of therapy used. However, certain inferences can be drawn from analysis of the data. Treatment with carbohydrate and insulin alone is unsatisfactory. It does not supply the elements that have been wasted by the body. Dissipation of saltless water by means of insensible perspiration and of salt and water through renal activity and vomiting continue. These processes lead to a concentration of all the solutes in the interstitial fluids. If the initial loss of salt is small, the concentrations of serum electrolytes may appear surprisingly normal unless the degree of body fluid loss is considered. Treatment with fluid without salt is also unsatisfactory. Dilution occurs, making the salt depletion even more apparent. In either instance, changes in the concentration of $\mathrm{CO}_{2}$ do not indicate any essential improvement in the patient's condition.

The $\mathrm{CO}_{2}$ may rise further when no fluid is given because the body fluids become more concentrated. Nevertheless, administration of fluid at least has the advantage of combating, to some extent, dehydration. In both cases increases of $\mathrm{CO}_{2}$ are derived only from base liberated by combustion of ketones, while the actual base content of the body must suffer loss through urine and vomitus.

Since sodium, the major basic ion of intestinal fluids, cannot enter cells, the rising osmolar concentration of extracellular fluids that occurs when no salt and no fluid is given, must force the cells to give up water. Atchley, Loeb, Richards, Benedict and Driscoll (5) showed that during development of the dehydration of diabetic acidosis intracellular as well as extracellular fluid is lost, so that further concentration is even more undesirable. If fluid without salt be given, the osmotic concentration of the extracellular fluids would diminish, forcing the cells to imbibe water or perhaps to lose base. Carried far enough this would lead to complete disruption of the internal environment.

The importance of replacing the salt and fluid lost from the body has already been discussed in a previous article (3) in relation to shock and to the continuance of the stupor and other clinical symptoms. The rapid 
recovery of Case 82529, without any change in the concentration of serum bicarbonate, under the influence of parenteral administration of normal sodium chloride solution, was cited.

Three cases have been cited in which salt and fluid in varying amounts produced electrolyte patterns that were distorted and yet were more in keeping with a normal internal environment than most of the patterns resulting from either of the other forms of therapy discussed. Hyperchloremia with a continuously low $\mathrm{CO}_{2}$ concentration is frequently observed. This is shown strikingly in Case 90339. After forty-seven hours the serum of the patient had a chloride concentration of $118 \mathrm{~m} . \mathrm{Eq}$. and a $\mathrm{CO}_{2}$ concentration of only $17.8 \mathrm{~m}$.Eq. The concentration of her serum base was, however, normal, $150.6 \mathrm{~m}$.Eq. That this distortion is caused by a dilution of the serum with sodium chloride solution has already been pointed out. In diarrhea of infants, in which base is lost in excess of chloride, Hartmann (6) has claimed that treatment with sodium chloride and water alone is unsatisfactory because it gives rise to hyperchloremia and still further depresses bicarbonate. However, the depression of bicarbonate caused by sodium chloride is not due to displacement of one acid by the other, but to dilution of the bicarbonate by chloride solution. Hoag and Marples (7) have further pointed out that hyperchloremia occurs only when the amounts of chloride solution given are too small to ensure an adequate flow of urine. The effect of renal activity is shown by Case 71954-3. During the first eight hours the serum chloride concentration rose from 98.5 to $106.6 \mathrm{~m}$.Eq., although the base concentration remained constant at $140 \mathrm{~m} . \mathrm{Eq}$. During this period the excretion of total base, $22.2 \mathrm{~m}$.Eq., almost equalled the excretion of chloride, $24.9 \mathrm{~m}$.Eq. Although his $\mathrm{CO}_{2}$ concentration at this time was only $14.3 \mathrm{~m}$.Eq. the patient was entirely asymptomatic. During the next sixteen hours $184.1 \mathrm{~m}$. Eq. of chloride were excreted with only 31.1 m.Eq. of base. Unfortunately the electrolyte concentrations were not determined at the end of this time.

The presence of an extremely small chloride excretion at the time when hyperchloremia exists in the serum is also illustrated by Case 60404 . Serum chloride concentration rose from 95.2 to $105 \mathrm{~m}$.Eq. in five hours. During this period $260 \mathrm{~m}$.Eq. of chloride were administered but only $8 \mathrm{~m}$.Eq. were found in $556 \mathrm{cc}$. of urine. Extraordinary serum chloride concentrations are seen after clinical recovery. In Case 23514 the final chloride concentration was $120.7 \mathrm{~m} . \mathrm{Eq}$. Hartmann and Smyth (8) found that in pyloric obstruction, when chloride became depleted, patients, in spite of the retention of abnormally large amounts of $\mathrm{CO}_{2}$, excreted acid urine and thus maintained a normal concentration of base in the body fluids. In recovery from diabetic acidosis, under administration of sodium chloride, the reverse obtains. Until a normal base concentration is restored, the excretion of chloride remains low, even when serum chloride has been driven to an exceedingly high level. 
Despite the low $\mathrm{CO}_{2}$ concentration and the existence of hyperchloremia, the patients are entirely free of symptoms, provided that fluid and base requirements have been satisfied. In no case has the actual minute volume of respiration been determined, but in all cases which have progressed to this point, all visible hyperpnea has disappeared, although the bicarbonate concentration may be still as low as that observed in patients who, during the development of acidosis, suffered from extreme air hunger. The maintenance of a low alveolar $\mathrm{CO}_{2}$ tension with constant $\mathrm{CO}_{2}$ production must, undoubtedly, necessitate increased breathing. However, even a 50 per cent reduction of $\mathrm{CO}_{2}$ tension, once established, requires only 100 per cent overventilation, a degree of hyperventilation that is hardly noticeable. Furthermore, during recovery the $\mathrm{CO}_{2}$ tension is rising. It would seem that the restitution of volume and ionic concentration of the body fluids is of prime importance, whereas, the replacement of individual anions to recreate the normal pattern is of secondary importance. This rearrangement may not occur until long after the disappearance of all untoward symptoms and reactions. Field (9) and others have asserted that the bicarbonate deficit itself inhibits glucose utilization and have advanced this as a reason for administration of bicarbonate. In physiologic or clinical studies no basis for such an assertion is to be found. It has been demonstrated that administration of acidifying salts does not impair oxidation of sugar (10). In fact, Haldane (11) and others claim that it has an accelerating action on such oxidations. In other diseases with comparable acidosis, for example, nephritis, carbohydrate combustion seems not to be affected. Furthermore, it has been observed in the present series that utilization of sugar during recovery from diabetic acidosis may advance to the appearance of hypoglycemia while bicarbonate is still greatly depleted. In patient 79156 the blood sugar fell in successive observations from 427 to 277 to $117 \mathrm{mgm}$. per cent with corresponding bicarbonate values of 3.2, 6.4 and $9.7 \mathrm{~m}$.Eq. During this period of 6.5 hours he received more than 80 grams of sugar. There can be no doubt that he was burning carbohydrate rapidly at the time of the second and third blood examinations, although the bicarbonate was still at levels usually associated with coma.

Hartmann and Darrow (12) have presented a more logical argument for bicarbonate administration. It is evident from the serum electrolyte patterns at the height of acidosis that the concentration of $S$ is, in many cases, diminished. The actual quantity of $S$ must, of course, be even more depleted by loss of body fluids. That hyperchloremia develops when sodium chloride solution is given in the attempt to replace this deficit has already been demonstrated. In order, then, to restore the normal electrolyte pattern as speedily as possible, administration of bicarbonate would seem advisable. However, dilution of the extra- 
cellular fluids with sodium bicarbonate solution alone would cause greater distortion of the pattern than would dilution with sodium chloride, because of the difference in concentration of chloride and bicarbonate found in normal serum. Hence, a combination of the two salts should be given. It has already been stated that the amounts of sodium bicarbonate that were given to patients in this series were too small to permit any adequate deductions.

To be effective, large amounts of this salt would have to be given. Administering the salt by mouth is usually impossible because of the frequent occurrence of nausea and vomiting. This necessitates discontinuing all oral feeding and resorting entirely to parenteral administration of fluid, carbohydrate, and salts. The time required to prepare a solution of sodium bicarbonate for intravenous use precludes its use until the patient should be well on the way towards recovery, if large amounts of fluid and sodium chloride are given. Cunningham and Darrow (13) have proposed a solution made by mixing suitable proportions of sodium bicarbonate and hydrochloric acid. This solution, however, contains but $30 \mathrm{~m}$.Eq. of sodium bicarbonate per liter, an amount too small to evoke an adequate effect. Hartmann and Senn (14) have proposed using normal sodium lactate solution in amounts varied according to the estimated needs of the individual. This solution provides a means of giving an adequate amount of base to combine eventually with $\mathrm{CO}_{2}$. However, the solution also is not immediately available because it must be given intravenously and, therefore, cannot be made in advance if nonspecific reactions are to be avoided.

Although it may be desirable to give sodium bicarbonate or a substitute, this salt will not furnish all the requirements of the depleted body fluids. Chloride has been lost in large amounts. The administration of large amounts of sodium chloride as early in the course of treatment as possible is quite essential in accelerating recovery and should not be delayed until a suitable bicarbonate solution can be prepared.

\section{SUMMARY}

The effect, on the recovery from diabetic acidosis, of administering varying amounts of fluid, sodium chloride, and sodium bicarbonate, has been discussed. Treatment with carbohydrate and insulin alone, or with the addition of water only is unsatisfactory. The giving of sodium chloride leads to rapid recovery, even though hyperchloremia develop. The individual appears to retain chloride in high concentration until the base concentration approaches normal. Chloride is then excreted in excess of base. Clinical improvement parallels the restoration of body fluid and total ionic concentrations rather than the replacement of individual ions. 


\section{BIBLIOGRAPHY}

1. Peters, J. P., Kydd, D. M., Eisenman, A. J., and Hald, P. M., J. Clin. Invest., 1933, xii, 377. The Nature of Diabetic Acidosis.

2. Peters, J. P., Wakeman, A. M., Eisenman, A. J., and Lee, C., J. Clin. Invest., 1929, vi, 517. Total Acid-Base Equilibrium of Plasma in Health and Disease. X. The Acidosis of Nephritis..

3. Peters, J. P., Kydd, D. M., and Eisenman, A. J., J. Clin. Invest., 1933, xii, 355. Serum Proteins in Diabetic Acidosis.

4. Lavietes, P. H., Kydd, D. M., and Peters, J. P., Unpublished studies.

5. Atchley, D. W., Loeb, R. F., Richards, D. W., Jr., Benedict, E. M., and Driscoll, M. E., J. Clin. Invest., 1933, xii, 297. On Diabetic Acidosis. A Detailed Study of Electrolyte Balances Following the Withdrawal and Reestablishment of Insulin Therapy.

6. Hartmann, A. F., Am. J. Dis. Child., 1928, xxxv, 557. Chemical Changes Occurring in the Body as the Result of Certain Diseases. I. The Effects of Diarrhea, Vomiting, Hydration and Oliguria on the AcidBase Balance of the Plasma of Infants with Mastoiditis.

7. Hoag, L. A., and Marples, E., Proc. Soc. Exper. Biol. and Med., 1929, xxvi, 374. Treatment of Dehydration of Diarrhea with Parenteral Fluids. 1. Effect on Acid-Base States of Blood.

8. Hartmann, A. F., and Smyth, F. S., Am. J. Dis. Child., 1926, xxxii, 1. Chemical Changes in the Body Occurring as the Result of Vomiting.

9. Field, H., Jr., Personal communication cited by Peters and Van Slyke, Quantitative Clinical Chemistry. 1. Interpretations. Williams and Wilkins Company, Baltimore, 1931, p. 170.

10. Gilchrist, M. L., Arch. Dis. Child., 1932, vii, 69. The Influence of Acidosis on Carbohydrate Metabolism.

11. Haldane, J. B. S., Lancet, 1924, i, 537. Experimental and Therapeutic Alterations of Human Tissue Alkalinity.

12. Hartmann, A. F., and Darrow, D. C., J. Clin. Invest., 1928, vi, 257. The Composition of the Plasma in Severe Diabetic Acidosis and the Changes Taking Place during Recovery.

13. Cunningham, R. D. M., and Darrow, D. C., Am. J. Dis. Child., 1931, xli, 1347. Preparation of a Solution of Sodium Bicarbonate and Sodium Chloride for Hypodermoclysis.

14. Hartmann, A. F., and Senn, J. E., J. Clin. Invest., 1932, xi, 337. Studies in the Metabolism of Sodium $r$-Lactate. 11. Response of Human Subjects with Acidosis to the Intravenous Injection of Sodium rLactate. 